\title{
Calcineurin overexpression regulates APP metabolism
}

\author{
Suruchi Utreja, Sean J. Miller, Aleister Saunders* \\ Department of Biology, Drexel University, Philadelphia, USA; ${ }^{*}$ Corresponding Author: Aleister.Saunders@drexel.edu, \\ suruchiu86@gmail.com, seanjmiller90@gmail.com,
}

Received 23 May 2013; revised 2 July 2013; accepted 11 July 2013

Copyright (C) 2013 Suruchi Utreja et al. This is an open access article distributed under the Creative Commons Attribution License, which permits unrestricted use, distribution, and reproduction in any medium, provided the original work is properly cited.

\section{ABSTRACT}

Alzheimer's disease (AD) is a complex neurodegenerative disease, pathologically characterized by the accumulation of $\beta$-amyloid peptide $(A \beta)$ and intraneuronal neurofibrillary tangles. Production of the $A \beta$ peptide by the regulated intra-membrane proteolysis of the $\beta$-amyloid precursor protein (APP) is a central event in AD. Cognitive decline observed in AD models is dependent on $A \beta$ generation. One of the downstream effects mediated by soluble $A \beta$ oligomers includes the hyperactivation of calcineurin (CaN), $\mathrm{a} \mathrm{Ca}^{2+}$ and calmodulin (CaM)-dependent, serinethreonine protein phosphatase. CaN is one of the major phosphatase associated with cognitive and neurodegenerative effects of $A \beta$. A specific isoform of CaN, CaNA $\beta$, is one of the most up-regulated mRNAs in brains of early stage $A D$ patients. While $A \beta$ 's ability to activate $\mathrm{CaN}$ has been well studied, not much is known about how increased levels of CaN in AD brain can contribute towards $A \beta$ generation. In this study we specifically investigate if increased levels of CaNA $\beta$ regulate APP metabolism. Our results demonstrate that increased CaNA $\beta$ expression leads to increased APP levels and increased proteolytic products of APP, including $A \beta$. The data suggest that upregulation of $\mathrm{CaN}$ levels could contribute to neurodegeneration observed in $A D$ by increasing levels of $A \beta$, potentially forming a pathogenic feed forward loop.

Keywords: Alzheimer's Disease; Calcineurin; APP; A $\beta ;$ FK506

\section{INTRODUCTION}

Alzheimer's disease (AD) is a progressive neurode- generative brain disorder characterized by global cognitive decline involving memory, orientation, judgment, and reasoning [1]. The characteristic neuropathological hallmarks of AD include the presence of neurofibrillary tangles, senile plaques, and massive loss of neurons primarily in cerebral cortex and hippocampus in the brain [2]. The neurofibrillary tangles are paired helical filaments containing hyperphosphorylated form of the microtubule binding protein Tau. Senile plaques are extracellular deposits composed of $\beta$-amyloid (A $\beta$ ) peptide [2]. The $\mathrm{A} \beta$ peptides are generated by proteolytic processing of thelarge, type-I transmembrane protein, $\beta$-amyloid precursor protein (APP) [3]. Initially, APP is cleaved by $\beta$-secretase (BACE) to produce $\operatorname{sAPP} \beta$ and a carboxy terminal fragment of 99 amino acids (CTF-99). Subsequently, CTF-99 is then cleaved by $\gamma$-secretase to produce $\mathrm{A} \beta$ and AICD (APP intracellular domain) [4]. Alternatively, nonamyloidogenic processing of APP can also occur. In this scenario, APP is initially cleaved by $\alpha$-secretase (ADAM $9,10,17)$ to produce $\operatorname{sAPP} \alpha$ and a CTF of 83 amino acids in length (CTF-83). This shorter CTF-83 is then cleaved by $\gamma$-secretase to produce $\mathrm{p} 3$ and AICD. Because $\mathrm{A} \beta$ is generated as part of the normal APP processing, deregulation of APP processing can alter the levels of A $\beta$ being produced. Excessive production and accumulation of the $\mathrm{A} \beta$ peptide plays a fundamental role in $\mathrm{AD}$ pathology. Therefore, APP proteolysis is considered a central event in the development of AD.

$\mathrm{A} \beta$ 's ability to activate Calcineurin $(\mathrm{CaN})$, a member of the serine/threonine protein phosphatase family, has been well studied [5-7]. Specifically, increased A $\beta$ levels perturb intracellular calcium $\left(\mathrm{Ca}^{2+}\right)$ levels which, in turn, lead to $\mathrm{CaN}$ hyperactivity. This $\mathrm{A} \beta$-mediated increase in $\mathrm{CaN}$ activity has been shown to play a role in altered synaptic plasticity, neuronal apoptosis, loss of dendritic spines, simplification of dendritic arborization and neuritic dystrophies, elevation in glutamate, excitotoxic cell death and behavioral impairments observed in some $\mathrm{AD}$ 
mouse models [6-10]. Pharmacologic inhibition of $\mathrm{CaN}$ activity, using FK506, has been shown to partially attenuate these effects.

$\mathrm{CaN}$ is a heterodimer consisting of a $58-64 \mathrm{kDa}$ catalytic subunit (CaNA) tightly bound to a $19 \mathrm{kDa}$ regulatory subunit (CaNB) [11]. CaNA is composed of a catalytic domain and a regulatory domain that contains a CaNB binding sequence, a calmodulin (CaM) binding domain and an autoinhibitory domain. The phosphatase is activated when $\mathrm{Ca}^{2+} / \mathrm{CaM}$ binds to it, triggering a conformational change that releases the autoinhibitory domain from catalytic active site [12]. CaNA can also be activated by proteolytic cleavage of the autoinhibitory domain, resulting in a $\mathrm{Ca}^{2+} / \mathrm{CaM}$-independent active phosphatase [13-16]. Three mammalian isoforms of CaNA $(\alpha$, $\beta, \gamma$ ) have been identified, and their amino acid sequences have been highly conserved between different organisms [17]. $\mathrm{CaNA} \alpha$ and $\mathrm{CaNA} \beta$ are ubiquitously distributed, while CaNA $\gamma$ is specifically expressed in the testis. The regulatory subunit $\mathrm{CaNB}$, has two mammalian isoforms CaNB1 and CaNB2, which are also highly conserved and have four $\mathrm{Ca}^{2+}$ binding "EF-hands" each. CaNB1 is ubiquitously expressed as a heterodimer with $\mathrm{CaNA} \alpha$ or $\beta$, while CaNB2 binds to CaNA $\gamma$ and is expressed only in testis [11].

$\mathrm{CaN}$ is widely expressed in mammalian tissues, but its concentration in brain is $10-20$ times higher than that found in other tissues [11]. In the brain, $\mathrm{CaN}$ accounts more than $1 \%$ of the total protein content $[18,19]$. Interestingly, levels of $\mathrm{CaNA} \beta$ have been reported to be significantly upregulated in the Alzheimer's disease hippocampus $[20,21]$. CaNA $\beta$ has also been shown to modulate tau phosphorylation state suggesting its role in development of neurofibrillary tangles [22]. In our study we investigate if CaNA $\beta$ overexpression, in HEK293 cells, alters levels of APP and its proteolytic products including $\mathrm{A} \beta$.

\section{EXPERIMENTAL PROCEDURES}

\subsection{Plasmids}

CaNA $\beta$ ( $P P P 3 C B)$ overexpression plasmid (pCMVSPORT6) was purchased from Open Biosystems. Swedish $\mathrm{APP}_{695}$ plasmid (pCMV-SPORT5) was generated in our lab by mutating $\mathrm{APP}_{695}$ (pCMV-SPORT5).

\subsection{Antibodies}

Primary antibodies utilized were: a polyclonal antibody raised to the C-terminus of APP (A8717; Sigma Aldrich, Inc), $\mathrm{C} 1 / 6.1$ monoclonal antibody recognizing the $\mathrm{C}$ terminus of APP was kindly provided by P. M. Mathews, an Anti-APP monoclonal antibody was used to detect levels of sAPP total (22C11, Millipore), a monoclonal anti- $\beta$-Actin antibody (A5441, Sigma Aldrich, Inc), a polyclonal antibody that recognizes APP phosphorylated at Thr668 (D90B8; Cell Signaling Technologies), a monoclonal antibody to the CaN catalytic subunit (C1956; Sigma, Inc).Secondary antibodies used were: goat antiRabbit IR-Dye800 CW (926-3211;LiCor Inc) and/or goat anti-Mouse IR Dye 680 (926-3200; LiCor Inc).

\subsection{Cell Culture and Overexpression Experiments}

HEK-293 cells were purchased from the American Type Culture Collection (ATCC) (Manassas, VA). HEK-293 cells were cultured in Dulbecco's modified Eagles medium (DMEM), supplemented with 10\% fetal bovine serum, penicillin $(25,000 \mathrm{U} / \mathrm{ml})$ and streptomycin $(25,000$ $\mu \mathrm{g} / \mathrm{ml})$. HEK293 cells were then transiently transfected with $\mathrm{CaNA} \beta$ overexpression construct and an appropriate empty vector control. 48 hours post-transfection, cell lysates were collected and used for Western Blot analysis. Conditioned media from the above experiment was used to detect s-APP total and $\mathrm{A} \beta$ levels.

\subsection{Western Blot Analysis}

After the treatments, cells were washed twice with cold PBS and then lysed in RIPA Buffer (50 mM Tris, $150 \mathrm{mM} \mathrm{NaCl}, 1 \%$ SDS, $1 \% \mathrm{NP}-40,0.5 \%$ deoxycholate, $\mathrm{pH}$ 8.0) containing HALT protease and phosphatase inhibitor cocktail (ThermoFisher). $10 \mu \mathrm{l}$ of the whole cell lysates were used to determine protein concentration (BCA Protein Assay Kit; Pierce, Inc.). Based on the protein concentrations, samples for Western Blot analysis were prepared using the $4 \times$ Nupage LDS sample buffer (InVitrogen, Inc.) containing $0.2 \%$ BME ( $\beta$-Mercaptoethanol, Sigma Aldrich). Equal concentrations of protein were loaded into each well of NuPAGE 4\% - 12\% Bis Tris Gel. A molecular weight ladder (Two color protein standard, 928-40001, Li-COR) was always run alongside of the experimental samples. Proteins were then transferred from the gel to a $0.25 \mu \mathrm{m}$ PVDF membrane (Millipore) using a semi-dry transfer apparatus. Blots were blocked (Blocking buffer, Li-COR) and then probed with primary and secondary antibodies. Target protein densitometry was quantified using the software (ODYSSEY Infrared imaging system, Application software) and normalized to the densitometry of $\beta$-actin. All Western blot experiments were performed in triplicates.

\subsection{RNA Extraction and Quantitative PCR}

48 hours post-transfection, cells were washed with cold PBS and total RNA was isolated using RNeasy Mini Kit (Qiagen Inc.). To quantify APP mRNA levels, cDNA was synthesized using total RNA, N6 random primers 
and SuperScript II Reverse Transcriptase (Invitrogen). cDNA was then diluted 1:15 using RNase free water and mixed with APP or GAPDH primer/probe sets (Applied Biosystems, Inc.; APP Catalog \# Hs00169098 m1; GAPDH Catalog \# Hs99999905_m1), 2× PCR Universal Master Mix (Applied Biosystems, Inc.) and amplified using an ABI 7500 Real Time PCR system following the manufacturer's directions. GAPDH was used as an internal control. To determine differences in APP mRNA levels, we utilized the $\Delta \Delta \mathrm{Ct}$ method and calculated fold change.

\subsection{A $\beta$ ELISA}

A $\beta$ levels were determined using commercially available Human/Rat $\beta$ Amyloid (40) ELISA Kit Wako (Wako Catalog \#294-62501, 96 tests) according to the manufacturer's instructions. Conditioned media obtained from the treatments were diluted 1:30 in manufacturer supplied sample buffer. Conditioned media was then used for measuring secreted $\mathrm{A} \beta_{40}$. In brief, $\mathrm{A} \beta_{40}$ levels $(\mathrm{pg} / \mathrm{ml})$ were quantified following the manufacturer's instructions.

\subsection{Statistical Analysis}

Values in the text and figures are presented as means \pm standard errors of at least three independent experiments. Equal variance or separate variance two-sample student's t-test were used, as appropriate, to compare two groups. “*” indicates $\mathrm{p}<0.05$, “**” indicates $\mathrm{p}<0.01$, and "****" indicates $\mathrm{p}<0.001$. "n.s." indicates $\mathrm{p}$-values greater than 0.05 .

\section{RESULTS}

\subsection{CaNA $\beta$ Overexpression Increases Levels of FL-APP, APP-CTF \& sAPP-Total and $A \beta_{40}$}

To test whether CaNA $\beta$ alters APP metabolism, CaNA $\beta$ was overexpressed in HEK293 cells (Figure 1(a)). The effect of overexpression on levels of endogenous, full-length $\mathrm{APP}_{770}$ and $\mathrm{APP}_{751}$ (FL-APP) and their proteolytic fragments was assessed. Upon CaNA $\beta$ overexpression, we observed a significant increase in levels of endogenous FL-APP levels (Figures 1(a) \& (b)) and APP-CTF's (CTF-83 and CTF-99) (Figures 1(c) \& (d)). Both CTF-83 and CTF-99 were significantly increased when they were quantified individually and normalized to $\beta$-actin (data not shown). To determine if this increase observed in levels of APP-CTF's was due to increase in FL-APP levels, we normalized APP-CTF levels to FLAPP levels and observed no significant change, suggesting that the increase we observe in APP-CTF's levels is solely due to the increase in FL-APP levels (Figure 1(e)).

Next, we wanted to determine if $\mathrm{CaNA} \beta$ overexpres-

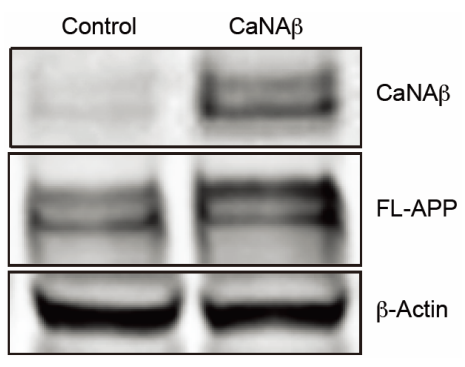

(a)

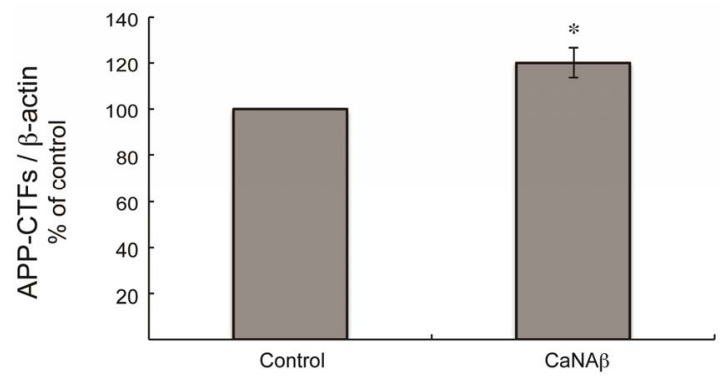

(d)

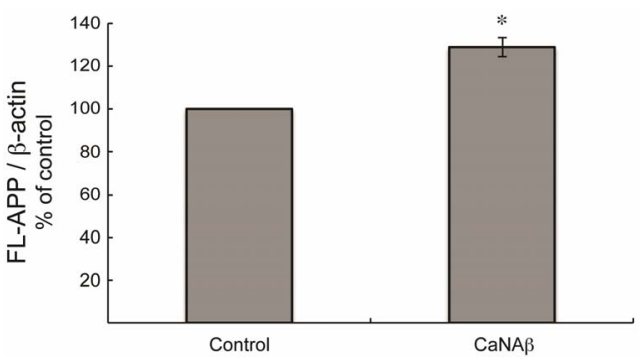

(b)

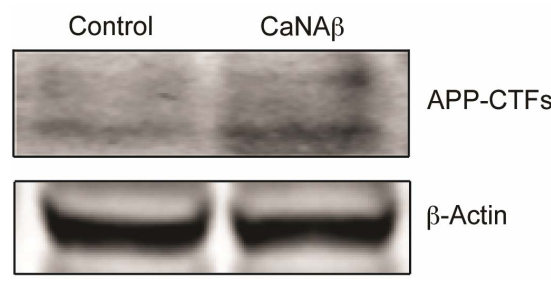

(c)

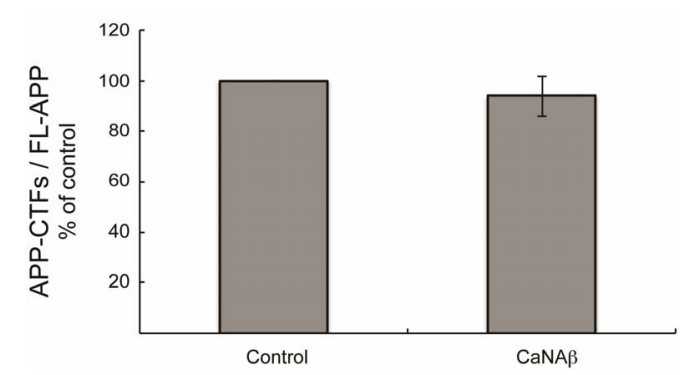

(e)

Figure 1. CaNA $\beta$ overexpression alters levels of endogenous APP and APP-CTF's. CaNA $\beta$ or an empty vector was transiently transfected into HEK293 cells. (a) Representative western blot showing an increase in levels of CaNA $\beta$ and endogenous FL-APP when $\mathrm{CaNA} \beta$ is overexpressed; (b) Quantification shows a significant increase in levels of endogenous FL-APP. $\beta$-Actin was used as a loading control; (c) A representative Western blot showing an increase in levels of endogenous APP-CTF's upon CaNA $\beta$ overexpression; (d) Quantification shows that the increase observed in levels of APP-CTF's is significant; (e) APP-CTF's levels were normalized to FL-APP levels and no significant difference in the ratio is observed. Values are means \pm standard error of 3 independent experiments. "*”: $\mathrm{p}<0.05$ and "n.s.": p-values $>0.05$ (Student's t-test). 
sion in HEK293 could alter levels of other APP proteolytic products. Therefore, CaNA $\beta$ was co-overexpressed with Swedish $\mathrm{APP}_{695}\left(\mathrm{APP}_{\mathrm{Swe}}\right)$ in HEK293 cells. Similar to what we observed with endogenous FL-APP, CaNA $\beta$ co-overexpression resulted in a significant increase in FL-APP ${ }_{\text {Swe }}$ levels (Figures 2(a) \& (b)) and in $\mathrm{APP}_{\mathrm{Swe}^{-}}$ CTF levels (CTF-83 and CTF-99) (Figures 2(c) \& (d)). Increase in both CTF-83 and CTF-99 was observed when normalized to $\beta$-actin (data not shown). Again the increase observed in $\mathrm{APP}_{\mathrm{Swe}}-\mathrm{CTF}$ 's levels was dependent on the increase in FL-APP ${ }_{\text {Swe }}$ levels (Figure 2(e)). Given that $\mathrm{CaNA} \beta$ overexpression lead to an increase in FL$\mathrm{APP}_{\text {Swe }}$ and $\mathrm{APP}_{\text {Swe }}-\mathrm{CTF}$ levels, next we used the conditioned media collected from these cells to probe for soluble APP levels ( sAPP $_{\text {Swe }}$ Total). We observed a significant increase in levels of $\mathrm{SAPP}_{\text {Swe }}$ total with $\mathrm{CaNA} \beta$ co- overexpression with Swedish $\mathrm{APP}_{695}$ (Figures 2(f) \& (g)). Lastly, we measured levels of $\mathrm{A} \beta_{40}$ in the conditioned media and observed a significant increase in levels of $\mathrm{A} \beta_{40}$ with $\mathrm{CaNA} \beta$ overexpression (Figure 2(h)). This increase in $\mathrm{A} \beta_{40}$ levels was again dependent on the increase in FL-APP ${ }_{\text {Swe }}$ levels (Figure 2(i)).

\subsection{CaNA $\beta$ Overexpression Increases Phospho-APP swe and Phospho-APP Swe $^{-C T F}$ Levels}

Given CaNA $\beta$ 's role as a phosphatase we investigated the effect of CaNA $\beta$ overexpression on APP phosphorylation. Co-overexpressing $\mathrm{CaNA} \beta$ and $\mathrm{APP}_{\text {Swe }}$ lead to a significant increase in phospho-Thr668 FL-APP Swe $_{\text {levels }}$ (Figures 3(a) \& (b)). A significant increase in phos-

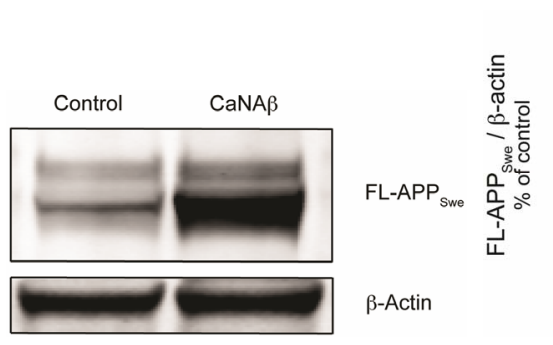

(a)

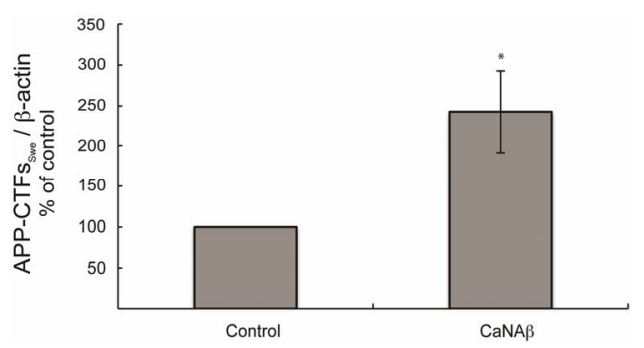

(d)

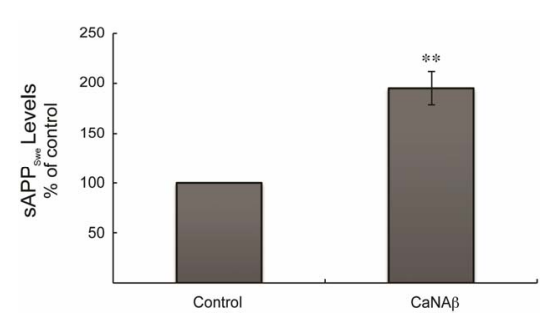

(g)

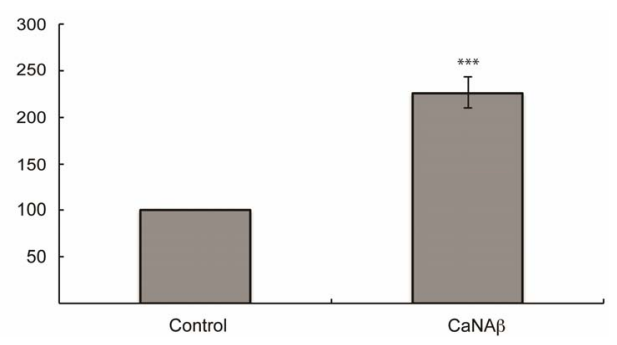

(b)

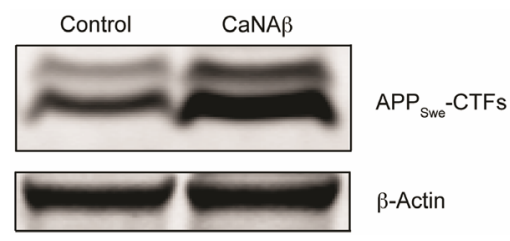

(c)

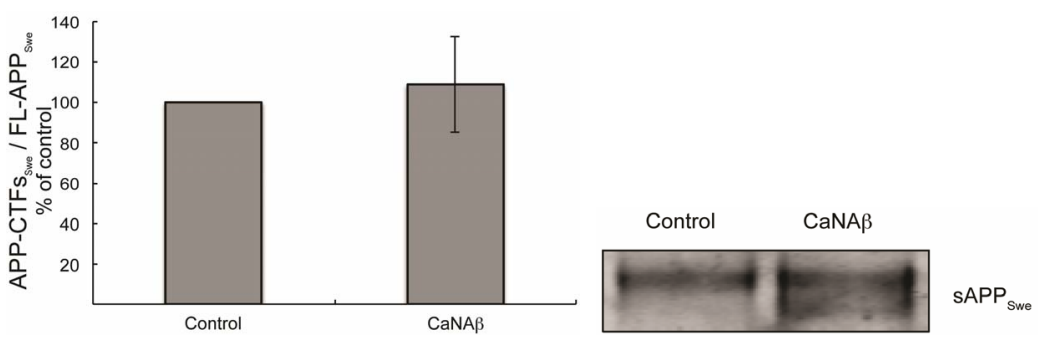

(e)

(f)

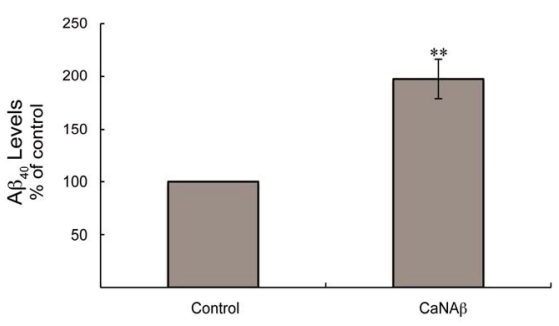

(h)

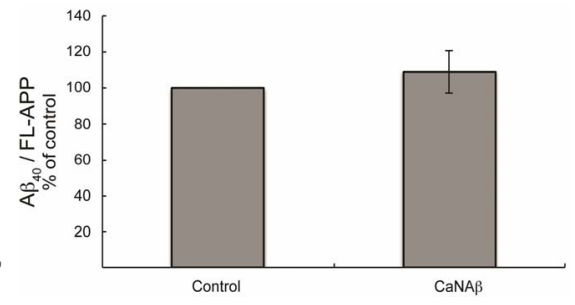

(i)

Figure 2. $\mathrm{CaNA} \beta$ co-overexpression with $\mathrm{APP}_{\mathrm{Swe}}$ alters levels of APP and APP proteolytic products. CaNA $\beta$ and $\mathrm{APP}$ Swe were co-overexpressed in HEK293 cells. An empty vector was also co-overexpressed with $\mathrm{APP}_{\mathrm{Swe}}$ plasmid as the control for the experi-

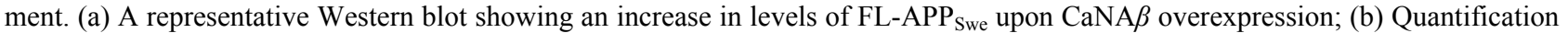

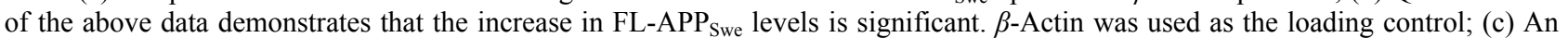
increase in levels of $\mathrm{APP}_{\mathrm{Swe}}-\mathrm{CTF}$ 's was also observed with CaNA $\beta$ overexpression; (d) Quantification of the above data shows that

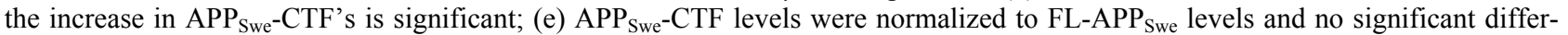
ence is observed; (f) Conditioned media from co-transfected cells was used to assess sAPP total levels; (g) Quantification of the sAPP total Western blot data shows a significant increase in levels of sAPP total with CaNA $\beta$ overexpression; (h) CaNA $\beta$ overexpression led to a significant increase in levels of A $\beta 40$ as assessed by ELISA assay; (i) A $\beta 40$ levels were normalized to levels of FL-APP and no significant difference is observed. Values are means \pm standard errors of 3 independent experiments. “*”: $p<0.05$, “**”: $\mathrm{p}<0.01$, “***”:p $<0.001$ and “n.s.": p-values $>0.05$ (Student's t-test). 


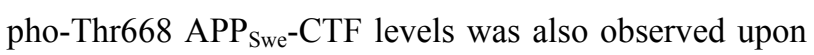
co-overexpression (Figures 3(d) \& (e)). In order to determine the proportion of FL-APP Swe $_{\text {that }}$ was phosphorylated on CaNA $\beta$ co-overexpression, we normalized levels of phospho-Thr668 FL-APP ${ }_{\text {Swe }}$ to total FL-APP ${ }_{\text {Swe }}$ and observed no significant change (Figure 3(c)). However, when phospho-Thr668 APP $_{\text {Swe }}$-CTF's were normalized to total $\mathrm{APP}_{\text {Swe }}-\mathrm{CTFs}$, a significant increase in the proportion of phospho-Thr668 $\mathrm{APP}_{\mathrm{Swe}}$-CTFs was observed (Figure 3(f)).

\subsection{CaNA $\beta$ Overexpression Does Not Alter APP MRNA Levels}

Since we observe an increase in levels of endogenous and exogenous FL-APP upon CaNA $\beta$ overexpression it is unlikely that $\mathrm{CaNA} \beta$ is altering APP expression via a transcriptional mechanism. It is possible that the mRNA stability could be affected. To assess both of these possibilities we utilized RT-QPCR to determine if $\mathrm{CaNA} \beta$ overexpression resulted in altered APP mRNA levels. As expected, overexpression of CaNA $\beta$ in HEK293 cells had no significant effect on endogenous APP mRNA levels suggesting that $\mathrm{CaNA} \beta$ alters APP levels posttranscriptionally (Figure 4).

\section{DISCUSSION}

In our study, we demonstrate that $\mathrm{CaNA} \beta$ overexpression can regulate APP metabolism. We have undertaken a systematic search for regulators of APP metabolism utilizing an APP-Gal4/Gal4-UAS luciferase reporter system [24-25]. Using this approach we identified $\mathrm{CaNA} \beta$ as a positional candidate gene that could function in regulating APP metabolism. Specifically, we observed that CaNA $\beta$ knockdown in SH-SY5Y APP-Gal4 cells that stably carry this reporter system, leads to a significant decrease in AICD-Gal4 mediated luciferase activity when compared to the non-silencing shRNA control. CaNA $\beta$, when overexpressed in the same cell line, led to a significant increase in AICD-Gal4 mediated luciferase activity compared to the appropriate empty vector (Utreja et al, submitted). Knockdown and overexpression of the $\mathrm{CaNA} \beta$ subunit resulted in consistent and opposite effects on APP proteolysis as measured by this assay, suggesting that $\mathrm{CaNA} \beta$ plays a reproducible role in APP metabolism regulation in this cellular model. Here, we have extended these initial studies by investigating whether CaNA $\beta$ expression alters other aspects of APP proteolysis.

Overexpression of $\mathrm{CaNA} \beta$ in HEK293 cells lead to a

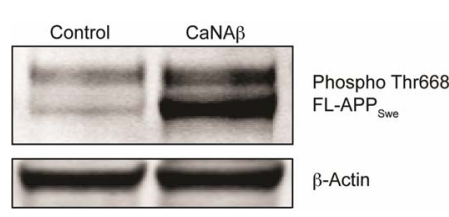

(a)

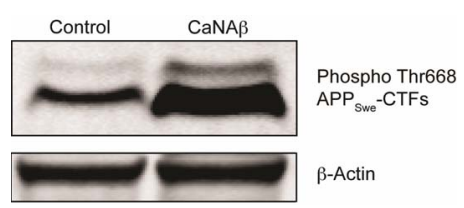

(d)

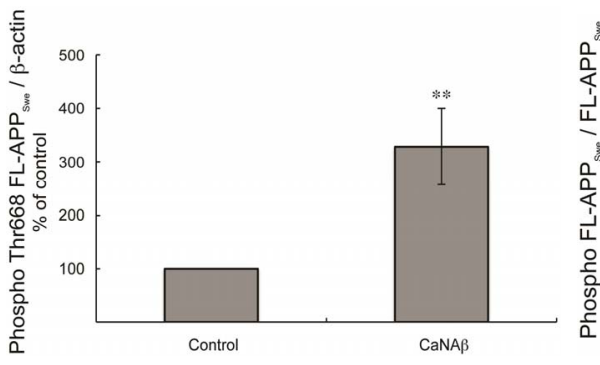

(b)

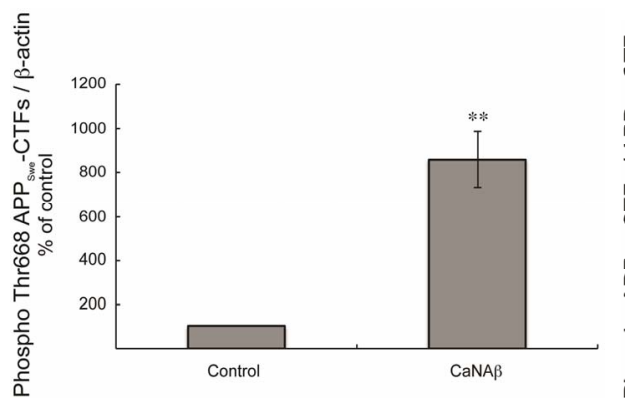

(e)

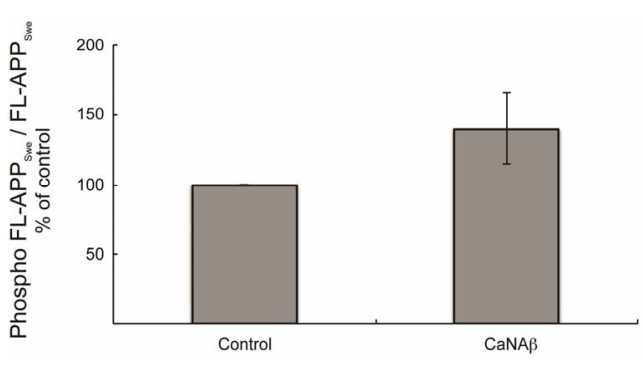

(c)

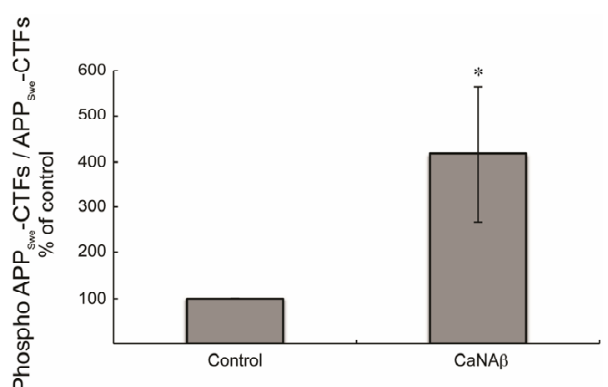

(f)

Figure 3. $\mathrm{CaNA} \beta$ co-overexpression with $\mathrm{APP}_{\mathrm{Swe}}$ leads to increase in Thr668 Phosphorylation (a) Representative western blot from the HEK293 cell lysates co-overexpressing $\mathrm{CaNA} \beta$ and $\mathrm{APP}_{\mathrm{Swe}}$; (b) Quantification of the above experiment shows an increase in levels of phospho-Thr668 FL-APP Swe $_{\text {; }}$ (c) When phospho-Thr668 FL-APP Swe $_{\text {levels were normalized to total FL-APP }}$ Swe levels, no significant difference is observed showing that this increase is proportional to the levels of FL-APP ${ }_{\text {Swe }}$; (d) Western blot showing an increase in levels of phospho-Thr668 $\mathrm{APP}_{\mathrm{Swe}}-\mathrm{CTF}$ 's on $\mathrm{CaNA} \beta$ overexpression; (e) Quantification of the above experiment reveals that the increase is significant; (f) Normalization of phospho-Thr668 APP Swe $_{-}-\mathrm{CTF}$ levels to total APP $\mathrm{Swe}_{\mathrm{STF}}-\mathrm{CT}$ shows a significant increase in the proportion $\mathrm{APP}_{\mathrm{Swe}}-\mathrm{CTF}$ that are phosphorylated at Thr668. 14 Values are means \pm standard errors of 3 independent experiments. “*”: $\mathrm{p}<0.05$, “**”: $\mathrm{p}<0.01$ and "n.s.": p-values $>0.05$ (Student's t-test). 


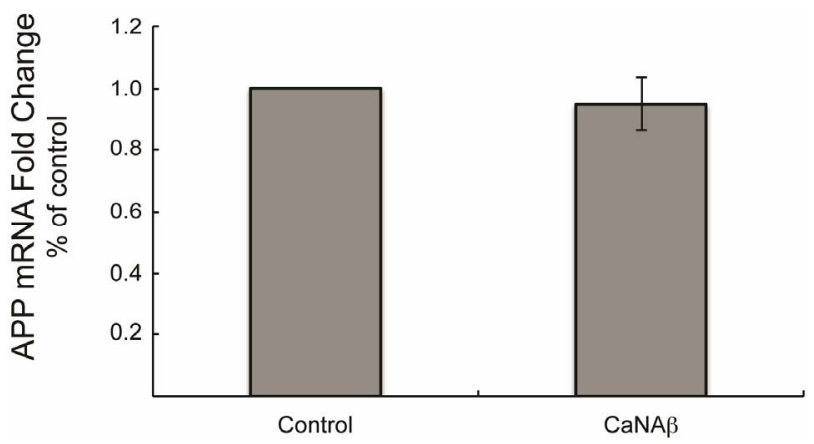

Figure 4. $\mathrm{CaNA} \beta$ overexpression does not alter APP mRNA levels No differences in fold change of endogenous APP mRNA is observed upon CaNA $\beta$ overexpression in HEK 293 cells, suggesting that $\mathrm{CaNA} \beta$ overexpression does not regulate APP transcription.

significant increase in levels of FL-APP, and APP-CTF's (CTF-83 and CTF-99), sAPP total and $\mathrm{A} \beta_{40}$ levels. This increase in FL-APP and APP-CTF's was observed with endogenous levels of APP and overexpressed APP Swe. $_{\text {. }}$ The increase in APP levels observed are most likely due to post-transcriptional effects as no change in APP mRNA levels was observed upon $\mathrm{CaNA} \beta$ overexpression. Given CaN's role as a serine/threonine specific phosphatase, we also examined affect of $\mathrm{CaNA} \beta$ expression on APP phosphorylation state. FL-APP and APP-CTF, which increased upon $\mathrm{CaNA} \beta$ overexpression, were found to be phosphorylated at Thr668 residue. When phosphoThr668 FL-APP and phospho-Thr668 APP-CTF's levels were normalized to total FL-APP and APP-CTF levels, respectively, no increase was observed in overall phosphorylation of FL-APP but a significant increase inphospho-Thr668 APP-CTF's was observed. Taken together, we conclude that increased expression of $\mathrm{CaNA} \beta$ can alter APP metabolism and lead to increased production of $\mathrm{A} \beta$, one of the main causes of Alzheimer's disease. Therefore, down regulating levels of $\mathrm{CaNA} \beta$ could be used as a potential therapeutic for decreasing $\mathrm{A} \beta$ levels.

$\mathrm{CaN}$ is an interesting and an important target in $\mathrm{AD}$ because of its critical role in learning and memory [11]. $\mathrm{CaN}$ being a serine/threonine specific phosphatase mediates the dephosphorylation of several cellular proteins and is involved in different signaling pathways. CaN mediated dephosphorylation of pCREB [25], pNFAT [26], p-PP1 [27,28], p-GSK-3 [29], and pBAD [30,31] could putatively explain its individual role in synaptic protein loss, neuroinflammation (neuronal and astrocytic), decreased neurotransmission, hyperphosphorylated tau, and cell death observed in AD models. However, the role of $\mathrm{CaN}$ in the brain may be very complicated. Overexpression of $\mathrm{CaN}$ in mice results in altered synaptic function and deficits in memory retention [32, 33]. Knockdown of CaN activity facilitates PKA-de- pendent long-term potentiation and increased learning and memory [33]. Yet, completely knocking out calcineurin in Purkinje cells or in the anterior cortex impairs postsynaptic long-term potentiation and worsens cognition [34]. In aged rats, upregulation of $\mathrm{CaN}$ activity negatively correlates with cognitive performance [27]. Conversely, downregulation of $\mathrm{CaN}$ activity using autoinhibitory peptide has shown to improve memory in rodents [33]. Transgenic Tg2576 mice (AD mouse model), which accumulate $\mathrm{A} \beta$ and develop cognitive impairments prior to plaque deposition, have high CaN activity in central nervous system (CNS). Treatment of these mice with $\mathrm{CaN}$ inhibitor FK506 improves memory function, indicating that $\mathrm{CaN}$ is part of the $\mathrm{A} \beta$ mediated memory loss pathway [35]. It has been shown that exogenous application of synthetic amyloid- $\beta$ or oligomeric aggregates to cultured cells leads to a rapid and sustained rise in $\mathrm{Ca}^{2+}$ levels along with increase in CaN activity [8]. Furthermore, in vitro evidence indicates that $\mathrm{A} \beta$ induces neuronal apoptosis through a CaN-dependent mechanism [36]. Taken together, these studies suggest that $\mathrm{CaN}$ is the major phosphatase involved in cognitive and neurodegenerative effects of $\mathrm{A} \beta$. However, the ability of $\mathrm{CaN}$ to alter $\mathrm{A} \beta$ levels has not been investigated thoroughly.

Previously, cDNA microarray analysis has shown that CaNA $\beta$ is the most up-regulated gene in brain lesions of the early stage of AD [20]. RT-PCR analysis from the same study also confirmed that $\mathrm{CaNA} \beta$ was generally upregulated in the hippocampus of the early stage of $\mathrm{AD}$ [20]. The exact mechanism for upregulation of $\mathrm{CaNA} \beta$ in the $\mathrm{AD}$ brain remains unknown. In our study we report that overexpression of $\mathrm{CaNA} \beta$ increases levels of FLAPP and its proteolytic products including $\mathrm{A} \beta$. Hence, this increase in levels of $\mathrm{CaNA} \beta$ observed in $\mathrm{AD}$ brains could account for the increase in $\mathrm{A} \beta$ levels and $\mathrm{A} \beta$ mediated neurodegenerative affects. Our results indicate that this increase in APP levels observed with CaNA $\beta$ overexpression is post-transcriptional. Possibly, $\mathrm{CaNA} \beta$ could alter APP protein synthesis or protein degradation affecting the levels of APP and APP proteolytic products. Also, an increase in APP-CTF's Thr668 phosphorylation was observed upon $\mathrm{CaNA} \beta$ overexpression. $\mathrm{CaN}$ being a phosphatase, it's intriguing to observe an increase in APPCTF phosphorylation on CaNA $\beta$ overexpression. This could suggest that either $\mathrm{CaNA} \beta$ overexpression does not account for or lead to a change in the overall phosphatase activity of $\mathrm{CaN}$, or $\mathrm{CaNA} \beta$ has an indirect effect on APP-CTF phosphorylation. There has been evidence suggesting that overexpression of $\mathrm{CaN}$ leads to dephosphorylation of GSK-3 $\beta$ at Ser-9, increasing its activity and leading to an increase in Tau phosphorylation [29]. Therefore it is possible that $\mathrm{CaNA} \beta$ could affect GSK- $3 \beta$ phosphorylation/activity leading to an increase in levels of APP-CTF phosphorylation at Thr668. Lastly, activity and 
levels of $\mathrm{CaN}$ in $\mathrm{AD}$ brains/AD models are differentially regulated. Specifically, $\mathrm{CaN}$ activity is globally reduced in $\mathrm{AD}$ brains and during aging $[37,38]$. Conversely, protein levels of $\mathrm{CaN}$ are inversely correlated with dementia severity [38]. Therefore it will be important to determine if increased levels of $\mathrm{CaNA} \beta$ leads to altered $\mathrm{CaN}$ activity.

To our knowledge our study is the first to show that CaNA $\beta$ overexpression regulates APP levels and proteolysis leading to an increase in $\mathrm{A} \beta$ levels. Results presented in our study provide an important link between clinically observed increase in $\mathrm{CaNA} \beta$ levels in $\mathrm{AD}$ brains and the experimentally observed increase in $\mathrm{A} \beta$ levels. The results suggest the presence of potentially pathogenic feed forward loop between $\mathrm{CaN}$ and $\mathrm{A} \beta$.

\section{ACKNOWLEDGEMENTS}

The authors would like to thank the members of the Saunders laboratory for helpful discussions. This work was support by grants from the National Institute of Neurologic Disease and Stroke (NS057295 to AJS).

\section{REFERENCES}

[1] Tanzi, R.E. and Bertram, L. (2005) Twenty years of the Alzheimer's disease amyloid hypothesis: A genetic perspective. Cell, 120, 545-555. doi:10.1016/j.cell.2005.02.008

[2] Selkoe, D.J. (2003) Aging, amyloid, and Alzheimer's disease: A perspective in honor of CarlCotman. Neurochemical Research, 28, 1705-1713. doi:10.1023/A:1026065122854

[3] Shoji, M., et al. (1992) Production of the Alzheimer amyloid beta protein by normalproteolytic processing. Science, 258, 126-129. doi:10.1126/science. 1439760

[4] De Strooper, B. and Annaert, W. (2000) Proteolytic processing and cell biological functions of theamyloid precursor protein. Journal of Cell Science, 113, 1857-1870.

[5] Kuchibhotla, K.V. et al. (2008) Abeta plaques lead to aberrant regulation of calcium homeostasis in vivo resulting in structural and functional disruption of neuronal networks. Neuron, 59, 214-225. doi:10.1016/j.neuron.2008.06.008

[6] Wu, H.Y., et al. (2010) Amyloid beta induces the morphological neurodegenerative triad of spine loss, dendritic simplification, and neuritic dystrophies through calcineurin activation. The Journal of Neuroscience, 30, 26362649. doi:10.1523/JNEUROSCI.4456-09.2010

[7] Loo, D.T., et al. (1993) Apoptosis is induced by betaamyloid in cultured central nervous system neurons. Proceedings of the National Academy of Sciences of the United States of America, 90, 7951-7955. doi:10.1073/pnas.90.17.7951

[8] Reese, L.C., Zhang, W., Dineley, K.T., Kayed, R. and Taglialatela, G. (2008) Selective induction of calcineurin activity and signaling by oligomeric amyloid beta. Aging

\section{Cell, 7, 824-835. doi:10.1111/j.1474-9726.2008.00434.x}

[9] Agostinho, P., Lopes, J.P., Velez, Z. and Oliveira, C.R., (2008) Overactivation of calcineurin induced by amyloid-beta and prion proteins. Neurochemistry International, 52, 1226-1233. doi:10.1016/j.neuint.2008.01.005

[10] Abdul, H.M., et al. (2009) Cognitive decline in Alzheimer's disease is associated with selective changes in calcineurin/NFAT signaling. The Journal of Neuroscience, 29, 12957-12969. doi:10.1523/JNEUROSCI.1064-09.2009

[11] Rusnak, F. and Mertz, P. (2000) Calcineurin: Form and function. Physiological Reviews, 80, 1483-1521

[12] Klee, C.B., Crouch, T.H. and Krinks, M.H. (1979) Calcineurin: A calcium- and calmodulin-binding protein of the nervous system. Proceedings of the National Academy of Sciences of the United States of America, 76, 6270-6273. doi:10.1073/pnas.76.12.6270

[13] Klee, C.B., Ren, H. and Wang, X. (1998) Regulation of the calmodulin-stimulated protein phosphatase, calcineurin. The Journal of Biological Chemistry, 273, 13367-13370. doi:10.1074/jbc.273.22.13367

[14] Perrino, B.A., Ng, L.Y. and Soderling, T. R. (1995) Calcium regulation of calcineurin phosphatase activity by its B subunit and calmodulin. Role of the autoinhibitory domain. The Journal of Biological Chemistry, 270, 7012.

[15] Wu, H.Y., et al. (2004) Critical role of calpain-mediated cleavage of calcineurin inexcitotoxic neurodegeneration. The Journal of Biological Chemistry, 279, 4929-4940. doi:10.1074/jbc.M309767200

[16] Mohmmad Abdul, H., Baig, I., Levine H., Guttmann, R.P. and Norris, C.M. (2011) Proteolysis of calcineurin is increased in human hippocampus during mild cognitive impairment and is stimulated by oligomeric Abeta in primary cell culture. Aging Cell, 10, 103-113. doi:10.1111/j.1474-9726.2010.00645.x

[17] Shibasaki, F., Hallin, U. and Uchino, H. (2002) Calcineurin as a multifunctional regulator. The Journal of Biochemistry, 131, 1-15. doi:10.1093/oxfordjournals.jbchem.a003063

[18] Klee, C.B., Draetta, G.F. and Hubbard, M.J. (1988) Calcineurin. Advances in Enzymology and Related Areas of Molecular Biology, 61, 149-200.

[19] Yakel, J.L. (1997) Calcineurin regulation of synaptic function: From ion channels to transmitter release and gene transcription. Trends in Pharmacological Sciences, 18, 124-134. doi:10.1016/S0165-6147(97)01046-8

[20] Hata, R., et al. (2001) Up-regulation of calcineurin Abeta mRNA in the Alzheimer's disease brain: Assessment by cDNA microarray. Biochemical and Biophysical Research Communications, 284, 310-316. doi:10.1006/bbrc.2001.4968

[21] Taguchi, K., et al. (2005) Identification of hippocampus-related candidate genes for Alzheimer's disease. Annals of Neurology, 57, 585-588. doi:10.1002/ana.20433

[22] Wei, Q., Holzer, M., Brueckner, M. K., Liu, Y. and Arendt, T. (2005) Dephosphorylation of tau protein by calcineurin triturated into neural living cells. Cellular and Molecular 
Neurobiology, 22, 13-24.

[23] Zhang, C., et al. (2007) An AICD-based functional screen to identify APP metabolism regulators. Molecular Neurodegeneration, 2, 15. doi:10.1186/1750-1326-2-15

[24] Zuchner, S., et al. (2008) Linkage and association study of late-ons et al zheimer disease families linked to 9p21.3. Annals of Human Genetics, 72, 725-731. doi:10.1111/j.1469-1809.2008.00474.x

[25] Bito, H., Deisseroth, K. and Tsien, R.W. (1996) CREB phosphorylation and dephosphorylation: $\mathrm{A} \mathrm{Ca}(2+)-$ and stimulus duration-dependent switch for hippocampal gene expression. Cell, 87, 1203-1214. doi:10.1016/S0092-8674(00)81816-4

[26] Abdul, H.M., et al. (2009) Cognitive decline in Alzheimer's disease is associated with selective changes in calcineurin/NFAT signaling. The Journal of Neuroscience, 29, 12957-12969. doi:10.1523/JNEUROSCI.1064-09.2009

[27] Foster, T.C., Sharrow, K.M., Masse, J.R., Norris, C.M. and Kumar, A. (2001) Calcineurin links Ca2+ dysregulation with brain aging. The Journal of Neuroscience, 21, 4066-4073.

[28] Mulkey, R.M., Endo, S., Shenolikar, S. and Malenka, R.C. (1994) Involvement of acalcineurin/inhibitor-1 phosphatase cascade in hippocampal long-term depression. $\mathrm{Na}$ ture, 369, 486-488. doi:10.1038/369486a0

[29] Kim, Y., et al. (2009) Calcineurin dephosphorylates glycogen synthase kinase- 3 beta atserine- 9 in neuroblast-derived cells. Journal of Neurochemistry, 111, 344-354. doi:10.1111/j.1471-4159.2009.06318.x

[30] Wang, H.G., et al. (1999) $\mathrm{Ca}^{2+}$-induced apoptosis through calcineurin dephosphorylation of BAD. Science, 284, 339343. doi:10.1126/science.284.5412.339

[31] Asai, A., et al. (1999) High level calcineurin activity predisposes neuronal cells to apoptosis. The Journal of Biological Chemistry, 274, 34450-34458. doi: $10.1074 /$ jbc. 274.48 .34450

[32] Kayyali, U.S., Zhang, W., Yee, A.G., Seidman, J.G. and Potter, H. (1999) Cytoskeletal changes in the brains of mice lacking calcineurin A alpha. Journal of Neurochemistry, 68, 1668-1678 (1997). doi:10.1046/j.1471-4159.1997.68041668.x

[33] Malleret, G., et al. (2001) Inducible and reversible enhancement of learning, memory, andlong-term potentialtion by genetic inhibition of calcineurin. Cell, 104, 675686.

[34] Zeng, H., et al. (2001) Forebrain-specific calcineurin knockout selectively impairs bidirectional synaptic plasticity and working/episodic-like memory. Cell, 107, 617 629. doi:10.1016/S0092-8674(01)00585-2

[35] Dineley, K.T., Hogan, D., Zhang, W.R. and Taglialatela, G. (2007) Acute inhibition of calcineurin restores associative learning and memory in Tg2576 APP transgenic mice. Neurobiology of Learning and Memory, 88, 217-224. doi:10.1016/j.nlm.2007.03.010

[36] Agostinho, P. and Oliveira, C.R. (2003) Involvement of calcineurin in the neurotoxic effects induced by amyloid-beta and prion peptides. European Journal of Neuroscience, 17, 1189-1196. doi:10.1046/j.1460-9568.2003.02546.x

[37] Ladner, C.J., Czech, J., Maurice, J., Lorens, S.A. and Lee, J.M. (1996) Reduction of calcineurin enzymatic activity in Alzheimer's disease: Correlation with neuropathologic changes. Journal of Neuropathology \& Experimental Neurology, 55, 924-931.

[38] Karch, C.M., Jeng, A.T. and Goate, A.M. (2013) Calcium phosphatase calcineurin influences tau metabolism. Neurobiology of Aging, 34, 374-386. doi:10.1016/j.neurobiolaging.2012.05.003 